\title{
Robust Moving Region Boundary Extraction Using Second Order Statistics
}

\author{
Astrit Rexhepi and Farzin Mokhtarian \\ Centre for Vision, Speech, and Signal Processing \\ School of Electronics and Physical Sciences \\ University of Surrey \\ Guildford GU2 7XH \\ United Kingdom \\ a.rexhepi@surrey.ac.uk, \\ f.mokhtarian@surrey.ac.uk \\ http://www.surrey.ac.uk
}

\begin{abstract}
This paper describes a novel method of extracting moving region boundaries from the frames of an image sequence by filtering information of the first temporal cooccurrence matrix using the corresponding matrix from the next frame. The method described in this paper does not make use of any threshold and it is very robust and efficient with respect to noise.
\end{abstract}

\section{Introduction}

Cooccurrence matrices, originally called gray-tone spatial dependency matrices, were introduced by Haralick et al. [1, who used them to define textural properties of images.

Let $I$ be an image whose pixel gray levels are in the range $[0, \ldots, 255]$. Let $\delta=$ $(u, v)$ be an integer-valued displacement vector; $\delta$ specifies the relative position of the pixels at coordinates $(x, y)$ and $(x+u, y+v)$. A spatial cooccurrence matrix $M_{\delta}$ of $I$ is a $256 \times 256$ matrix whose $(i, j)$ element is the number of pairs of pixels of $I$ in relative position $\delta$ such that the first pixel has gray level $i$ and the second one has gray level $j$. Any $\delta$, or set of $\delta$-s, can be used to define a spatial cooccurrence matrix. In what follows we will usually assume that $\delta$ is a set of unit horizontal or vertical displacement, so that $M_{\delta}$ involves counts of pairs of neighboring pixels.

In addition to their original use in defining textural properties, cooccurrence matrices have been used for image segmentation. Ahuja and Rosenfeld [2] observed that pairs of pixels in the interiors of smooth regions in $I$ contribute to elements of $M_{\delta}$ near its main diagonal; thus in a histogram of the gray levels of the pixels that belong to such pairs, the peaks associated with the regions will be preserved, but the valleys associated with the boundaries between the regions will be suppressed, so that it becomes easier to select thresholds that separate the peaks and thus segment the image into the regions. In 3, Haddon and Boyce observed that homogeneous regions in $I$ give rise to peaks (clusters of 
high-valued elements) near the main diagonal of $M_{\delta}$, while boundaries between pairs of adjacent regions give rise to smaller peaks at off-diagonal locations; thus selecting the pixels that contribute to on-diagonal and off-diagonal peaks provides a segmentation of $I$ into homogeneous regions and boundaries. The peaks that can be expected to occur in cooccurrence matrices will be further described in Section 2.3 (compare [3]).

Pairs of pixels in the same spatial position that have a given temporal separation in a sequence of images can be used to define temporal cooccurrence matrices. Let $I$ and $J$ be images acquired at times $t$ and $t+d t$; thus $d t$ is the temporal displacement between $I$ and $J$. A temporal cooccurrence matrix $M_{d t}$ is a $256 \times 256$ matrix whose $(i, j)$ element is the number of pairs of pixels in corresponding positions in $I$ and $J$ such that the first pixel has gray level $i$ and the second one has gray level $j$.

Boyce et al. 4] introduced temporal cooccurrence matrices and used them in conjunction with spatial cooccurrence matrices to make initial estimates of the optical flow in an image sequence. They demonstrated that an initial probability of a pixel being in the interior or on the boundary of a region that has smooth optical flow in a given direction in a pair of images could be derived from the positions of the peaks in a spatial cooccurrence matrix of one of the images for a displacement in the given direction, and in the temporal cooccurrence matrix of the pair of images. Borghys et al. [5] used temporal cooccurrence matrices to detect sensor motion in a moving target detection system by comparing the spatial cooccurrence matrix of one of the images with the temporal cooccurrence matrix of the pair of images.

The following is the organization of this paper: Section 2 describes the peaks (clusters of high values) that can be expected to occur in spatial and temporal cooccurrence matrices when the image contains smooth regions. Section 3 describes our new method of extracting moving region boundaries from an image sequence by suppressing information from first temporal cooccurrence matrix using the corresponding matrix from the next frame. In Sections 4 and 5 we propose and develop a filter design and a feed-back system for moving boundary enhancement and noise removal. Section 6 describes the extension of our method for static boundary detection. Section 7 summarizes our work and discusses possible extensions.

\section{The Structure of Cooccurrence Matrices}

In the next section we will describe methods of using temporal cooccurrence matrices to extract moving region boundaries from the images of a sequence. In this section we describe the peak structures that should be present in spatial and temporal cooccurrence matrices.

We assume that an image $I$ is composed of regions in which (ignoring noise) the gray levels vary smoothly, and that if two regions are adjacent, they meet along a boundary at which the gray level changes significantly. It is well known (see [3]) that in a spatial cooccurrence matrix of $I$, each region (say having 


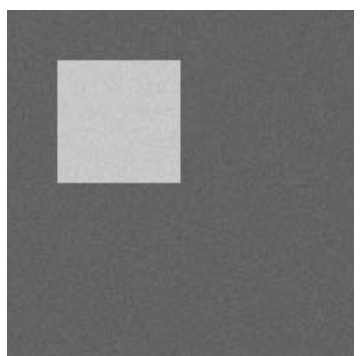

(a)

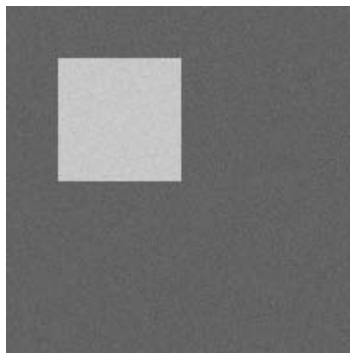

(b)

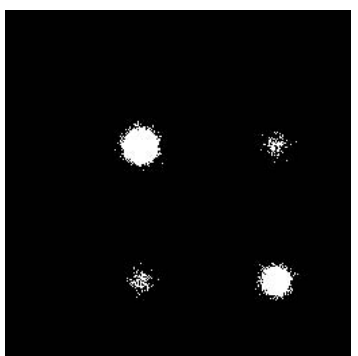

(c)

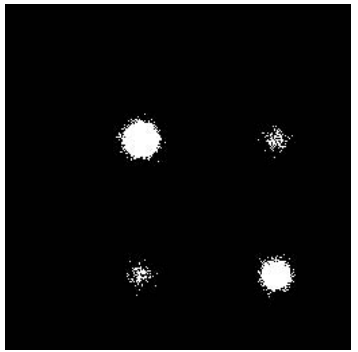

(d)

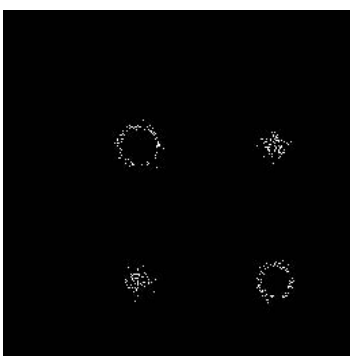

(e)

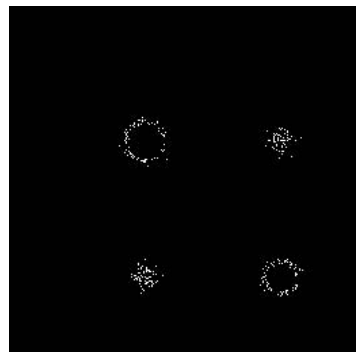

(f)

Fig. 1. (a and b) Two synthetic images(images I and J). (c and d) Their corresponding temporal cooccurrence matrices. (e and f) Their mutual suppression.

mean gray level $g$ ) should give rise to a peak centered on the main diagonal in approximate position $(g, g)$; the sum of the element values in this cluster should be proportional to the area of the region. Similarly, each boundary between two adjacent regions (say having mean gray levels $g$ and $h$ ) should give rise to a pair of off-diagonal peaks at approximate positions $(g, h)$ and $(h, g)$, and with value sum proportional to the length of the border. The same holds for temporal cooccurrence matrices when no motion occur, namely, we simply can assume that the whole frame is moving in the amount of one pixel. Thus, we can slide one of the frames in the amount of one pixel (top-down and left-right composed shift) and find the temporal cooccurrence matrices (in which ignoring border effects it is the same as spatial cooccurrence matrix),

Figure 17 is a test image $I$ containing a solid square with gray level 200 on a background with gray level 100. This image is composed with noise having a normal distribution with mean value zero and variance equal four. Let $J$ be another image(Figure 1b), the same as $I$ but where the solid square has moved from its original position by one pixel, and having the same instance of noise generated by a random generator.

We can treat $I$ and $J$ as consecutive frames of an image sequence acquired by a stationary camera. In this case the frames show an object (solid square) moving against a stationary background at a rate of one pixel per frame. In the temporal cooccurrence matrix of $I$ and $J$ (Figure 1 ), pairs of pixels that are in a moving region in both images will contribute to an on-diagonal peak. Similar, 
pairs of pixels that are in the background in both images will contribute also to another on-diagonal peak. Pairs of pixels that are covered up or uncovered by the motion will contribute to a pair of off-diagonal peaks.

\section{Extracting Boundaries Using Cooccurrence Matrices}

As discussed in Section 2, motion of an object against a contrasting background between two frames of an image sequence gives rise to off-diagonal peaks in a temporal cooccurrence matrix of the two frames. Thus it should be possible in principle to extract moving boundaries from a pair of successive frames of an image sequence by detecting off-diagonal peaks in the temporal cooccurrence matrix of the two frames and identifying the pixels in either of the frames that contributed to those peaks.

Unfortunately, off-diagonal peaks are not always easy to detect in cooccurrence matrices. Since the images are noisy, all the elements near the diagonal of a cooccurrence matrix tend to have high values, and the presence of these values makes it hard to detect off-diagonal peaks in the matrix that lie close to the diagonal since these peaks tend to have lower values. If we knew the standard deviation of the image noise, we could estimate how far the high values which are due to noise extend away from the diagonal of the cooccurrence matrix, and we could then look for peaks in the matrix that are farther than this from the diagonal; but information about the image noise level is usually not available.

In this section we describe a simple method of suppressing clusters of highvalued elements from a temporal cooccurrence matrix. As we will see, the suppressed matrix elements tend to lie near the diagonal of the matrix. Hence when the suppression process is applied to a temporal cooccurrence matrix the image pixels that contributed to the unsuppressed elements of the matrix tend to lie on the boundaries of moving regions.

Our method of suppressing clusters of high-valued elements from a cooccurrence matrix takes advantage of two observations:

(1) The matrix elements in the vicinity of a high-valued cluster almost certainly have nonzero values, so that the nonzero values in and near the cluster are "solid". On the other hand, it is more likely that there are zero-valued elements in and near a cluster of low-valued elements, so that the nonzero values in and near such a cluster are "sparse".

(2) As we saw in Section 2, the on-diagonal clusters in a cooccurrence matrix, which arise from regions in the image, can be expected to be symmetric around the main diagonal, and the off-diagonal clusters, which arise from motion, can be expected to occur in pairs whose means are symmetrically located around the main diagonal, since the noise in the image has zero mean. Hence if we have two cooccurrence matrices that are transposes of one another (see below), the clusters in these matrices should occur in the same approximate positions. 


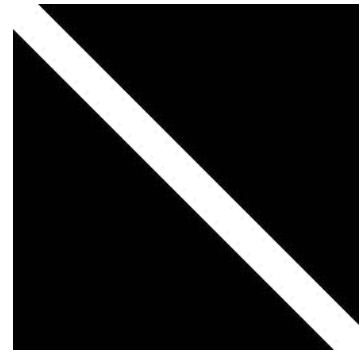

(a)

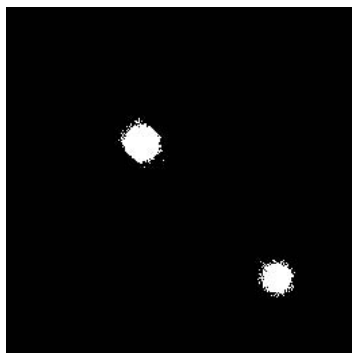

(b)

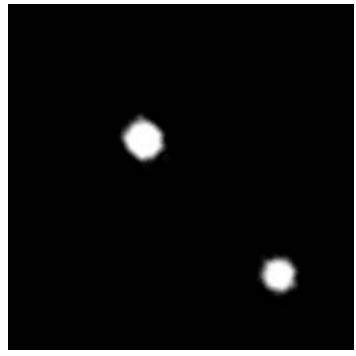

(c)

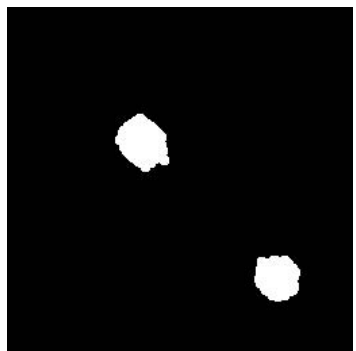

(d)

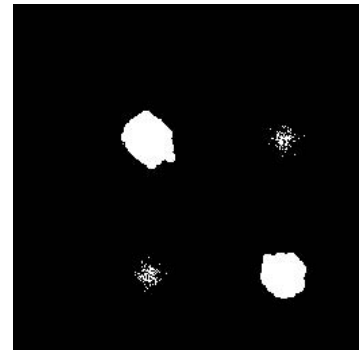

(e)

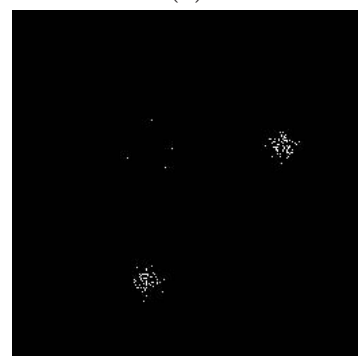

(f)

Fig. 2. (a) Filter F. (b) M+F. (c) Smoothed M+F. (d) Setting to one all the nonzero elements of the smoothed $M+F$ yielding $F^{\prime}$. (e) $M+F^{\prime}=M^{\prime}$. (f) $N / M^{\prime}$.

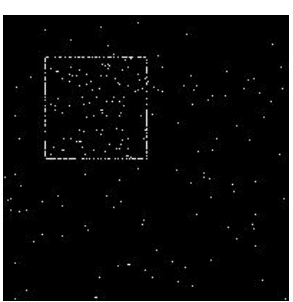

(a)

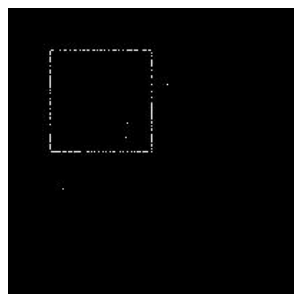

(b)

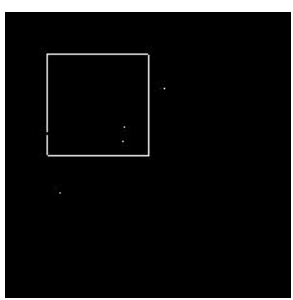

(c)

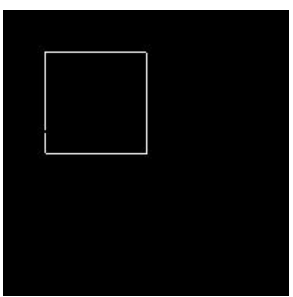

(d)

Fig. 3. (a) The result obtained by only Temporal Cooccurrence Matrices Suppression. (b) The result after filtering with F'. (c) The result after applying feedback-system. (d) The result after noise removal.

We can obtain temporal cooccurrence matrices that are transposes of one another by using reverse temporal displacements; i.e., if $I$ and $J$ are successive frames of an image sequence, we can use the temporal cooccurrence matrices of $I$ and $J$ (Figure 1 ) and of $J$ and $I$ (Figure 11). Evidently, Figure 15 and Figure 11d are transposes of each other.

Let $M$ and $N$ be two cooccurrence matrices that are transposes of one another. We suppress from $M$ all elements that are nonzero in $N$ (or vice versa). Elements of $M$ that are in or near a "solid" cluster will almost certainly have nonzero values in $N$; hence these elements will almost certainly be suppressed from $M$. On the 
other hand, many of the elements of $M$ that are in or near a "sparse" cluster will have zero values in $N$ because the nonzero elements of these clusters in $M$ and $N$ are not in exactly symmetrical positions; hence many of these elements will not be eliminated by the suppression process.

Figure 10 shows the nonzero elements of Figure 1 that are zero in Figure 1d, and Figure 1f shows the nonzero elements of Figure 11d that are zero in Figure 1k. We see that the "solid" parts of the matrix have been suppressed and the "sparse" parts have survived. Figure 3a shows the pixels of Figure 13 that contributed to the nonzero elements in Figure 11. Almost all of these pixels lie on region boundaries in Figure 3 3.

\section{The Problem of Residuals}

As we saw in the previous section, using temporal cooccurrence matrices suppression we achieved some important results. Namely, we are now able to detect moving boundaries and we don't need to set any threshold to do this, which is in contrast with existing methods. It is obvious that at this stage the results we just obtained contain some spurious noise pixels (Figure 3 a), but as we will show in this section, these spurious noise pixels we are able to eliminate successfully by developing some filters in a logical sense.

There are two reasons why these noise pixels appear in Figure [3a:

a) The on-diagonal clusters in temporal cooccurrence matrices are results of regions (static and inside moving objects regions). Thus, a homogenous region $R$ having an area (in pixels) $A$ will yield an on-diagonal cluster in the temporal cooccurrence matrix whose shape is circular centered at $(k, k)$ where $k$ is the mean value of $R$, and a radius $r$ that depends on noise variance (which is unknown) and the area of $R$. Usually, the noise has normal (multinomial) distribution or it can be approximated by normal distribution, thus, this cluster will be denser near $(k, k)$ and the density decreases as we go away from $(k, k)$. Places where the density of the cluster is low will survive suppression and we will call them residuals that have a ring shape as seen in Figure 10 and Figure 1f. These residuals appear as noise pixels in Figure 3 a.

b) Small regions having a unique gray level will yield (approximately) ondiagonal sparse clusters in the temporal cooccurrence. Hence, when suppression is applied most of the elements of these clusters will survive. These elements appear as noise in Figure 3 a.

\subsection{Designing the Filter to Suppress Residuals}

One way of suppressing residuals (and this is the only one we show in this paper) is to develop a filter $F$ as below:

Let $M$ and $N$ be the temporal cooccurrence matrices of $I$ and $J$ as shown in Figure 1 and Figure 11, and let $S$ be one of suppressed cooccurrence matrices, we put a filter-like-strip along the main diagonal in $M$ whose width is calculated using the following logic: 
Starting from the width equal to zero we increase it until the sum of nonzero elements of $S$ that do not belong inside the filter $F$ is equal(a place where the number of nonzero elements of $M$ that do not belong inside $F$ changes from being higher to smaller) the number of nonzero elements of $S$ that belongs inside the filter $F$. After we find $F$ (Figure 2a), the next task is to use this filter and place along the main diagonal of $N$. Nonzero elements of $N$ belonging inside the filter $F$ (Figure 2b) we smooth using an averaging filter-like-a-disk (or we can perform dilation instead) with radius 3 for all cases (Figure 2r), let us set all the nonzero elements of this matrix to one and denote it by $F^{\prime}$ as shown in Figure2 $\mathrm{d}$. Now, if we set to one all nonzero elements of this processed $N$ (Figure 22) and suppress $M$ from $N$ (Figure 2: ) the shape-like-a-ring residuals almost completely have disappeared, so let us denote it with $S^{\prime}$. The corresponding pixels of $I$ and $J$ that contributed to nonzero elements of $S^{\prime}$ are shown in Figure 3b, so let us denote it by $B^{\prime}$. It is obvious that Figure $3 \mathrm{~b}$ is almost noiseless compared to Figure 3 .

\section{$5 \quad$ Moving Boundaries Enhancement and Noise Removal}

In the previous section we were able to reduce the noise pixels using filter $F$. The results obtained are impressive, but further processing still has to be done because moving boundaries appear to be broken and there are still some noise pixels to be cleaned. In this section we will be dealing with the above mentioned problems.

Broken boundaries in Figure $3 \mathrm{~b}$ are result of suppression process. As discussed in Section 3, the off-diagonal clusters of $M$ and $N$ are results of moving boundaries. These clusters are sparse but it doesn't mean that when the suppression process is applied they will completely survive, something is possible to be suppressed. These suppressed elements in turn result in missing parts of boundaries in Figure 3b. To recover these missing parts of boundaries we will develop a feedback system that is based on the following assumption:

The density of nonzero elements in $B^{\prime}$ in and around boundaries is higher so that around nonzero elements of $B^{\prime}$ it is very likely to find elements (in the same spatial position) in $I$ and $J$ that contribute to nonzero elements of off-diagonal clusters of $M / F^{\prime}$ where / is a difference operator. On the other hand the density of nonzero elements of $B^{\prime}$ representing noise is very sparse, so that it is quite unlikely that around these points of $B^{\prime}$ we will find (in the same spatial position) elements in $I$ and $J$ that contribute to any of nonzero elements in $M / F^{\prime}$. Thus, principally it would be possible to recover suppressed elements of off-diagonal clusters of $S^{\prime}$ by searching in the neighborhood of nonzero elements of $B^{\prime}$. To do this, we developed a system as following:

- Smooth $B^{\prime}$ with an averaging filter of size $3 \times 3$.

- Develop $B^{\prime \prime}$ by setting to one the positive elements of smoothed $B^{\prime}$.

- Create images $I^{\prime}=I \diamond B^{\prime \prime}$ and $J^{\prime}=J \diamond B^{\prime \prime}$.

- Find their temporal cooccurrence matrix $M^{\prime}$. 
- Find $T=\left(M^{\prime} / F^{\prime}\right)$.

- Find elements of $I$ and $J$ that contributed to nonzero elements of $T$, yielding updated $B^{\prime}$.

- Repeat above steps until there is no change between consecutive $B^{\prime}$ images.

where $\diamond$ stands for element-by-element multiplication. Usually the last condition is satisfied in the third iteration, and the corresponding results we obtain (Figure 3.) are very satisfactory. Finally, the remaining noise points we reduce by using an operator of size $5 \times 5$ and exclude positive elements (belonging to the center of the operator) of updated $B^{\prime}$ if the total sum of positive elements inside the operator is less than 2. The result is shown in Figure 3 d, as we can see; the updated $B^{\prime}$ contains only the boundary of moving object. It might have been thought that the same results could have been achieved by using only $M / F$ or by only using image difference and use the width of $F$ as a threshold ( a number that decides what is the boundary and what noise), but it is not possible in general because low contrast boundaries will be vanished and many noise clusters will be left which are not possible to be cleaned in image domain because of their high density.

\section{Detecting Static Boundaries}

Our method can be extended for static boundary detection. To do this, we simply can assume that the whole frame is moving in the amount of one pixel. Thus, we can slide one of the frames in the amount of one pixel (top-down and left-right composed shift) and find the temporal cooccurrence matrices (in which ignoring border effects it is the same as spatial cooccurrence matrix), after we do this we filter on-diagonal elements using filter $F^{\prime}$ (in a similar way we did for moving boundaries in the previous sections) and find the corresponding pixels in $I$ and $J$ that contributed to the nonzero elements of their filtered temporal cooccurrence matrix.

Examples are given for real images that include both moving and static boundaries (Figure 4, Figure 5, Figure 6, and a comparison to image subtraction in Figure 7) (please observe threshold values we needed to take in order to get clean moving boundaries for the case of image subtraction, and compare results taken without any threshold using our system in 4 and 5 respectively).

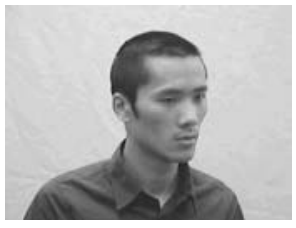

(a)

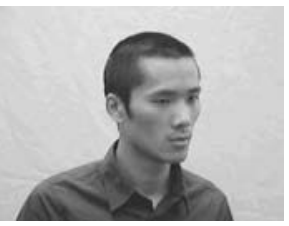

(b)

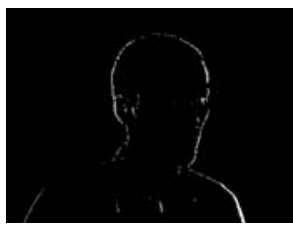

(c)

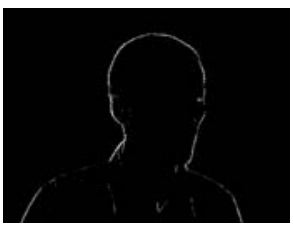

(d)

Fig. 4. (a) and (b): Two successive frames of an image sequence showing a moving man. (c) and (d); Moving and static boundary detection using our system. 


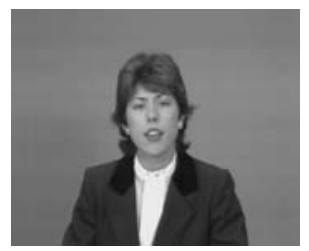

(a)

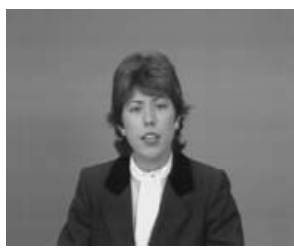

(b)

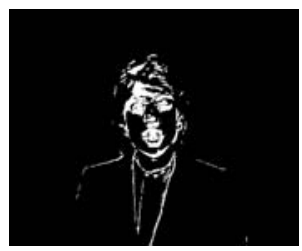

(c)

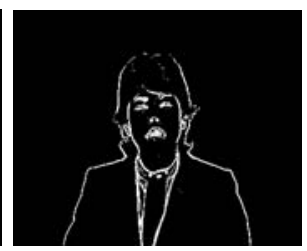

(d)

Fig. 5. (a) and (b): Two successive frames of an image sequence showing a moving speaker-woman. (c) and (d); Moving and static boundary detection using our system.

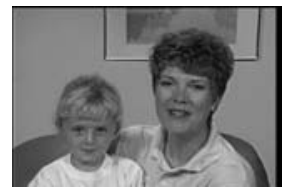

(a)

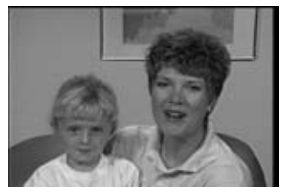

(b)

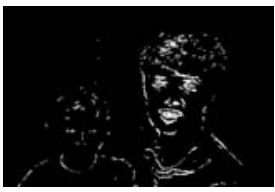

(c)

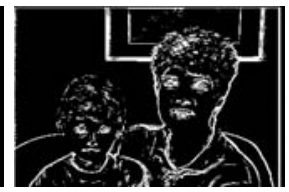

(d)

Fig. 6. (a) and (b): Two successive frames of an image sequence showing a moving woman and a child. (c) and (d); Moving and static boundary detection using our system.

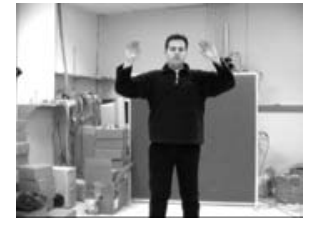

(a)

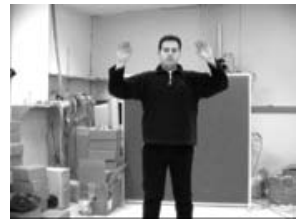

(b)

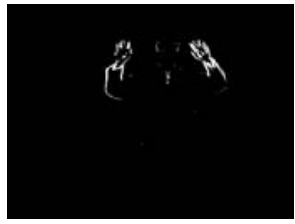

(c)

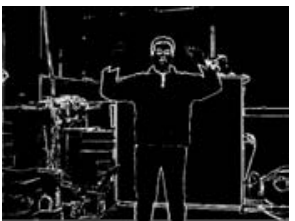

(d)

Fig. 7. (a) and (b): Two successive frames of an image sequence showing a man waiving his hands and arms. (c) and (d); Moving and static boundary detection using our system.

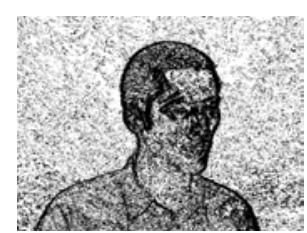

(a)

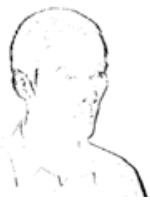

(b)

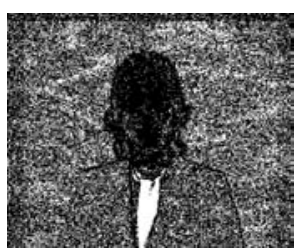

(c)

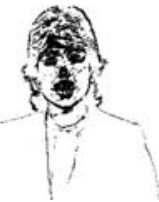

(d)

Fig. 8. (a): Image Subtraction of Figures 4 (a and b). (b): Moving boundaries after applying a threshold $T=40$. (c): Image Subtraction of Figures 5(a and b). (d) Moving boundaries after applying a threshold $T=7$. 


\section{Conclusions}

Moving Boundaries detection is a crucial step in computer vision that determines success or failure of the whole system. In this paper we presented an integrated system for moving boundary detection and its extension for static boundaries. This system is full-automatic (no human intervention is need) which has the following important properties:

- Does not make use of any threshold.

- Almost completely removes noise.

- In the same framework we can extract both moving and static boundaries.

- The complete system can be developed using only Boolean algebra.

- It is fast and very easy to realize even in hardware.

\section{References}

1. Haralick, R.M., Shanmugam, R., Dinstein, I.: Textural Features for Image Classification. IEEE Trans. on Systems, Man, and Cybernetics 3, 610-621 (1973)

2. Ahuja, N., Rosenfeld, A.: A Note on the Use of Second-order Gray-level Statistics for Threshold Selection. IEEE Trans. on Systems, Man, and Cybernetics 8, 895-898 (1978)

3. Haddon, J.F., Boyce, J.F.: Image Segmentation by Unifying Region and Boundary Information. IEEE Trans. on Pattern Analysis and Machine Intelligence 12, 929-948 (1990)

4. Boyce, J.F., Protheroe, S.R., Haddon, J.F.: A Relaxation Computation of Optic Flow from Spatial and Temporal Cooccurrence Matrices. International Conference on Pattern Recognition 3, 594-597 (1992)

5. Borghys, D., Verlinde, P., Perneel, C., Acheroy, M.: Long-range Target Detection in a Cluttered Environment Using Multi-sensor Image Sequences. Proc. SPIE 3068, 569-578 (1997) 